\title{
Haar Wavelet Matrices for the Numerical Solutions of Differential Equations
}

\author{
Sangeeta Arora \\ Research Scholar \\ Punjab Technical University \\ Jalandhar
}

\author{
Yadwinder Singh Brar \\ Department of ECE \\ Guru Nanak Dev Engineering \\ College Ludhiana
}

\author{
Sheo Kumar \\ Department of Mathematics \\ Dr. B.R. Ambedkar NIT \\ Jalandhar
}

\begin{abstract}
Haar Wavelets has become important tool for solving number of problems of science and engineering. In this paper a computational scheme is implemented using Haar matrices to find the numerical solution of differential equations with known initial and boundary conditions. We also presented exact solution, numerical solution and absolute error. Numerical experiments presented here are comparable with the available data. The algorithm used in this is very simple and easy to implement.
\end{abstract}

\section{Keywords}

Haar wavelets, Haar functions, Operational matrix, Differential equation.

\section{INTRODUCTION}

It has been observed from the literature that many researchers are developing fast and accurate numerical schemes to handle the different problems arising in various fields of science and engineering. In the past finite element methods and finite difference methods were commonly used for solving such problems. Nowadays wavelet methods are extensively applied to the problems for numerical solutions as wavelets methods have several advantages over FEM and FDM. . FEM is one of the successful and dominant numerical methods in last century. Wavelet analysis is a new technique that can be performed in several ways, a continuous wavelet transform, a discretized continuous wavelet transform, and a true discrete wavelet transform. With the rapid development of computer technology in the past few decades a broad range of numerical methods have been developed for different types of problems and achieved a great success like Haar wavelets methods. Haar wavelet method is simple and possesses less computational cost. In comparison with existing numerical schemes used to solve the PDE's, the Haar wavelet methods is an improvement over other methods in terms of accuracylt is extensively used in modeling and simulation of engineering and science due to its versatility and flexibility.

A wavelet is a mathematical function used to divide a given function or continuous-time signal into different scale components. The word wavelet is due to Morlet and Grossmann. In the early 1980s they used the French word ondelette, meaning "small wave". Soon it was transferred to English by translating "onde" into "wave", giving "wavelet". The study of wavelets has attained the present growth due to mathematical analysis of wavelets by Stromberg [1], Grossmann and Morlet and Meyer [2,3]. The concept of Multiresolution Analysis (MRA) was introduced by Mallat and Meyer [4]. Daubechies in 1988 presented a method to construct wavelets with compact support and scale functions [5].
A review of the basic properties of the wavelets and the decomposition and the reconstruction of functions in terms of the wavelet bases is given by Strang [6]. Many families of wavelets have been proposed in the literature. If one wants to use wavelets for the solution of differential equations, one therefore has to choose one specific family which is most advantageous for the intended application. Within one family there are also members of different degree. All these wavelet families can be classified as either being an orthogonal or biorthogonal family. Each orthogonal wavelet family is characterized by two functions- the mother scaling function and the mother wavelet. With a solid historical as well as practical background, Among the wavelet families, which are defined by an analytical expression, special attention deserves the Haar wavelets.

In 1910, Alfred Haar introduced the notion of wavelets. The Haar wavelet transform is one the earliest examples of what is known now as a compact, dyadic, orthonormal wavelet transform. Haar wavelets are made up of pairs of piecewise constant functions and are mathematically the simplest among all the wavelet families. A good feature of the Haar wavelets is the possibility to integrate them analytically arbitrary times. The Haar wavelets are very effective for treating singularities, since they can be interpreted as intermediate boundary conditions.

Haar wavelets are easy to handle from the mathematical aspect. Haar wavelets are very effective for solving ordinary differential and partial differential equations. Therefore the idea, to apply Haar wavelet technique was quite popular [7-10]. One property of the Haar wavelet is that it has compact support, which means that it vanishes outside of a finite interval.

In this paper, we apply Haar wavelet matrices to solve ordinary differential equations with initial or boundary condition known and we compare the results of numerical and exact solutions. This has been designed to promote the study of wavelets to beginners. Thus, simplified calculations are presented with necessary basic knowledge of haar functions and their generation.

\section{HAAR WAVELET MATRICES}

The Haar wavelet transform is the first known wavelet and was proposed in 1909 by Alfred Haar. A haar wavelet is a system of square wave having the first curve $h_{0}$ known as scaling function

$$
h_{0}(x)= \begin{cases}1 & \text { if } 0 \leq x \leq 1 \\ 0 & \text { otherwise }\end{cases}
$$

and second curve is $h_{1}$ is given by

$$
h_{1}(x)=\left\{\begin{array}{rc}
1, & 0 \leq x<\frac{1}{2} \\
-1, & \frac{1}{2} \leq x<1 \\
0 & \text { otherwise }
\end{array}\right.
$$


This is also known as mother wavelet. To perform the wavelet transform, haar wavelets uses translation and dilation using.

$$
h_{n}(x)=h_{1}\left(2^{j} x-k\right), n=2^{j}+k, j \geq 0,0 \leq k<2^{j}
$$

Moreover, for any square integrals function $\mathrm{y}(\mathrm{x})$ approximation can be made using the haar functions as

$$
y(x)=\sum_{i=1}^{2 m} a_{i} h_{i}(x)
$$

Where $h_{n}(x)=\left[h_{0}(x), h_{1}(x),,,,, h_{m-1}(x)\right]$. To express the haar matrix, we used the general notation as

$$
H_{m}=\left[h_{m}(1 / 2 m), h_{m}(3 / 2 m),,,, h_{m}(2 m-1 / 2 m)\right] .
$$

Thus we have

$$
\begin{gathered}
H_{1}=(1), H_{2}=\left(\begin{array}{cc}
1 & 1 \\
1 & -1
\end{array}\right) \\
H_{4}=\left(\begin{array}{cccc}
1 & 1 & 1 & 1 \\
1 & 1 & -1 & -1 \\
1 & -1 & 0 & 0 \\
0 & 0 & 1 & -1
\end{array}\right)
\end{gathered}
$$

$$
H_{8}=\left(\begin{array}{cccccccc}
1 & 1 & 1 & 1 & 1 & 1 & 1 & 1 \\
1 & 1 & 1 & 1 & -1 & -1 & -1 & -1 \\
1 & 1 & -1 & -1 & -1 & -1 & -1 & -1 \\
0 & 0 & 0 & 0 & 1 & 1 & -1 & -1 \\
1 & -1 & 0 & 0 & 0 & 0 & 0 & 0 \\
0 & 0 & 1 & -1 & 0 & 0 & 0 & 0 \\
0 & 0 & 1 & -1 & 0 & 0 & 0 & 0 \\
0 & 0 & 0 & 0 & 0 & 0 & 1 & -1
\end{array}\right)
$$

Integration over the vector $h_{m}$ is given by

$$
h_{m}=\int_{0}^{x} h_{m}(t) d t \approx P_{m} h_{m}(x), x \in[0,1]
$$

Operational matrix $P_{m}$ obtains the values as

$$
\begin{gathered}
P_{1}=\left(\frac{1}{2}\right), \\
P_{2}=\frac{1}{4}\left(\begin{array}{cc}
2 & -1 \\
1 & 0
\end{array}\right), \\
P_{4}=\frac{1}{16}\left(\begin{array}{cccc}
1 & 1 & 1 & 1 \\
1 & 1 & -1 & -1 \\
1 & -1 & 0 & 0 \\
0 & 0 & 1 & -1
\end{array}\right)
\end{gathered}
$$

$$
P_{3}=\left(\begin{array}{cccccccc}
32 & -16 & -8 & -8 & -4 & -4 & -4 & -4 \\
16 & 0 & -8 & 8 & -4 & -4 & 4 & 4 \\
4 & 4 & 0 & 0 & -4 & 4 & 0 & 0 \\
4 & 4 & 0 & 0 & -4 & 4 & 0 & 0 \\
1 & 1 & 2 & 0 & 0 & 0 & 0 & 0 \\
0 & 1 & -2 & -1 & 0 & 0 & 0 & 0 \\
0 & -1 & 0 & -1 & 0 & 0 & 0 & 0 \\
0 & -1 & 0 & 0 & 0 & 0 & 0 & 0
\end{array}\right)
$$

\section{NUMERICAL METHOD}

In this section, we present some numerical examples that are considered to apply the solution procedure presented in the previous section. Basis functions considered here are family of Haar wavelets. In the first example we solve the equation $y^{\prime \prime}+y=e^{x}$

With $y(0)=0, y^{\prime}(0)=0$ exact solution for this case is given by

$$
y(x)=\frac{1}{2}\left(e^{x}-\cos x-\sin x\right)
$$

To start the solution procedure, we have

$$
y(x)=\sum_{n=1}^{2 m} a_{i} h_{i}(x)
$$

Integrate both the sides and put the values of $y^{\prime}(0)=0$ and we found the values of $y^{\prime}(x)$, again integrate the side and put the value and we get the

$$
y(x)=\sum_{i=1}^{2 m} a_{1} p_{2, i}(x)
$$

after putting the values of $y^{\prime \prime}+y=e^{x}$ in

$$
\sum_{i=1}^{2 m} a_{i} h_{i}(x)+\sum_{i=1}^{2 m} a_{i} p_{2, i}(x)=e^{x}
$$

We found after simplification of this equation we get the result

$$
\sum_{i=1}^{2 m} a_{i}\left[h_{i}(x)+p_{2, i}(x)\right]=e^{x}
$$

then find and put the values of $a_{i}$ then we have $y(x)$ after putting the values of $y^{\prime \prime}(x)=y(x)=e^{x}$ we obtain numerical solution then we compare with the exact solution and we have the absolute error.

Table 1. Comparison of Exact and Haar Solution

\begin{tabular}{|c|c|c|c|}
\hline $\mathbf{x}$ & Exact Solution & Haar Solution & Absolute Error \\
\hline 0.125 & 0.0081 & 0.0088 & 0.0006 \\
\hline 0.375 & 0.0791 & 0.0810 & 0.0019 \\
\hline 0.625 & 0.2361 & 0.2399 & 0.0031 \\
\hline 0.875 & 0.4952 & 0.4992 & 0.0041 \\
\hline
\end{tabular}


If we take more collocation points and we will get more accurate results. Graphical representation of exact numerical solution is shown below.

\section{Graphical Representation of first example}

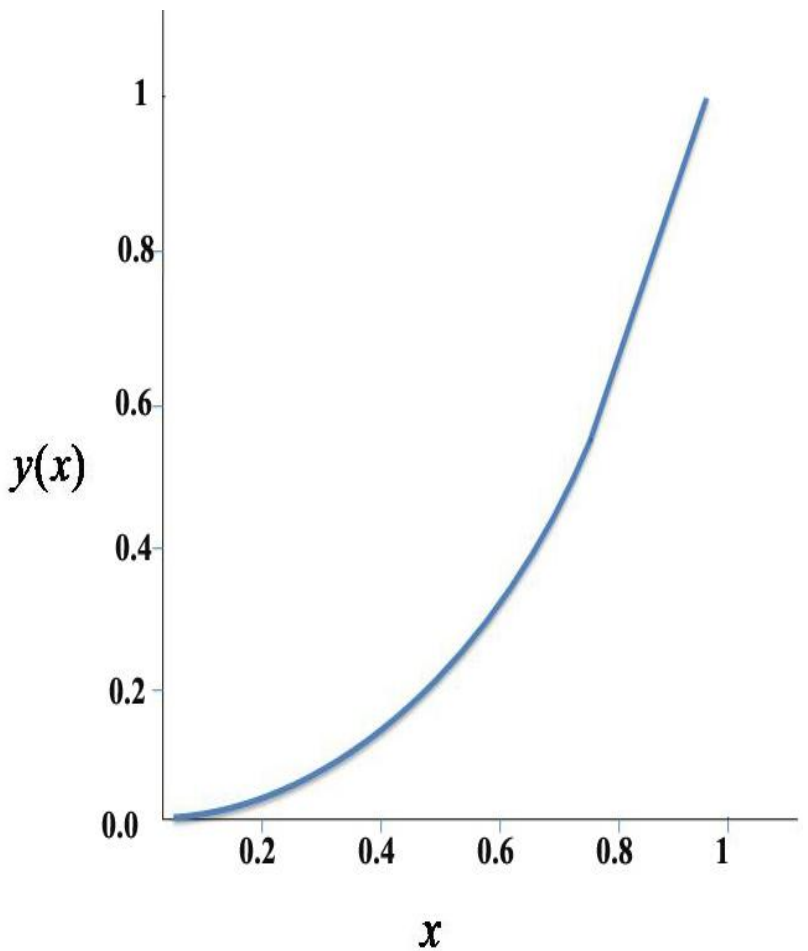

Figure 1(A): Exact Solution

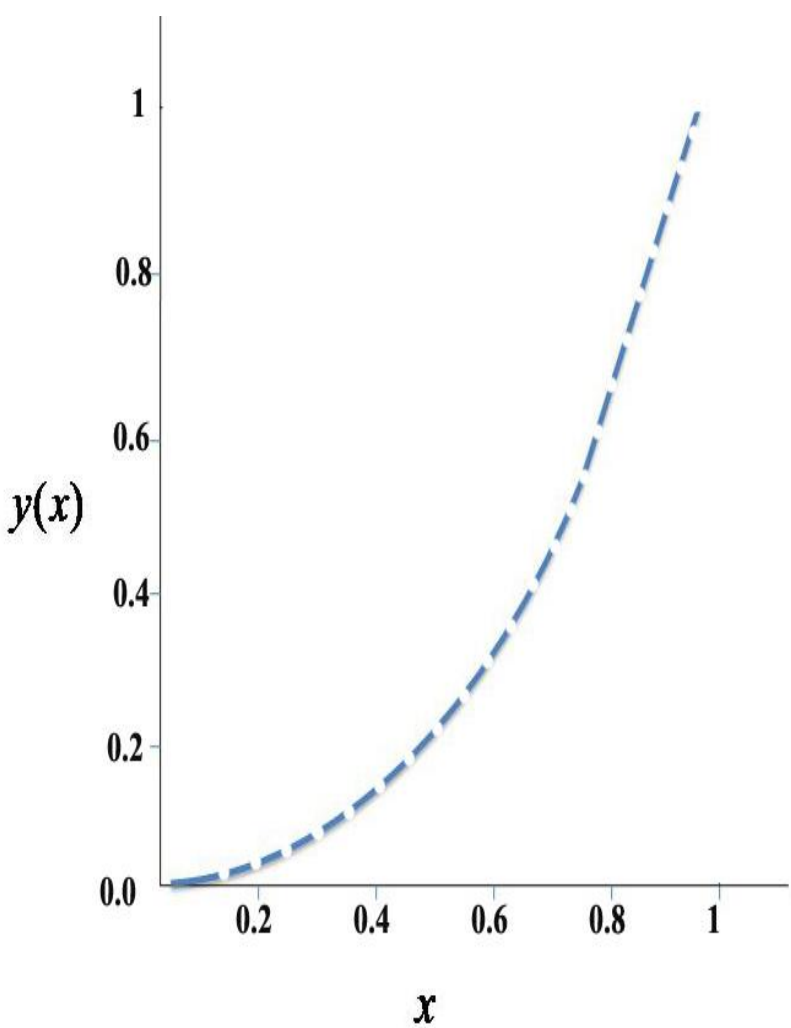

Figure 1(B) : Haar Solution
Another Example we will take

$$
-y^{\prime \prime}-y+x^{2}=0,0<x<1
$$

With $y(0)=0, y(1)=0$ and exact solution for this equation is

$$
y(x)=\frac{\sin (x)+2 \sin (1-x)}{\sin (1)}+x^{2}-2
$$

For numerical solution of above example we will take

$$
y(x)=\sum_{n=1}^{2 m} a_{i} h_{i}(x)
$$

after solving this we get

$$
y(x)=x+\sum_{i=1}^{2 m} a_{1}\left(p_{2, i}(x)-x p_{1, i}(1)\right)
$$

substitute the values in the given equation, we obtained numerical solution and compared with exact Solution.

Table 2. Comparison of Exact and Haar Solution

\begin{tabular}{|c|c|c|c|}
\hline $\mathbf{x}$ & Exact Solution & Haar Solution & Absolute Error \\
\hline 0.125 & -0.0119 & -0.0121 & 0.2159 \\
\hline 0.375 & -0.0334 & -0.0340 & 0.5210 \\
\hline 0.625 & -0.0435 & -0.0440 & 0.5022 \\
\hline 0.875 & -0.0259 & -0.0261 & 0.2023 \\
\hline
\end{tabular}

Graphical Representation of Second example

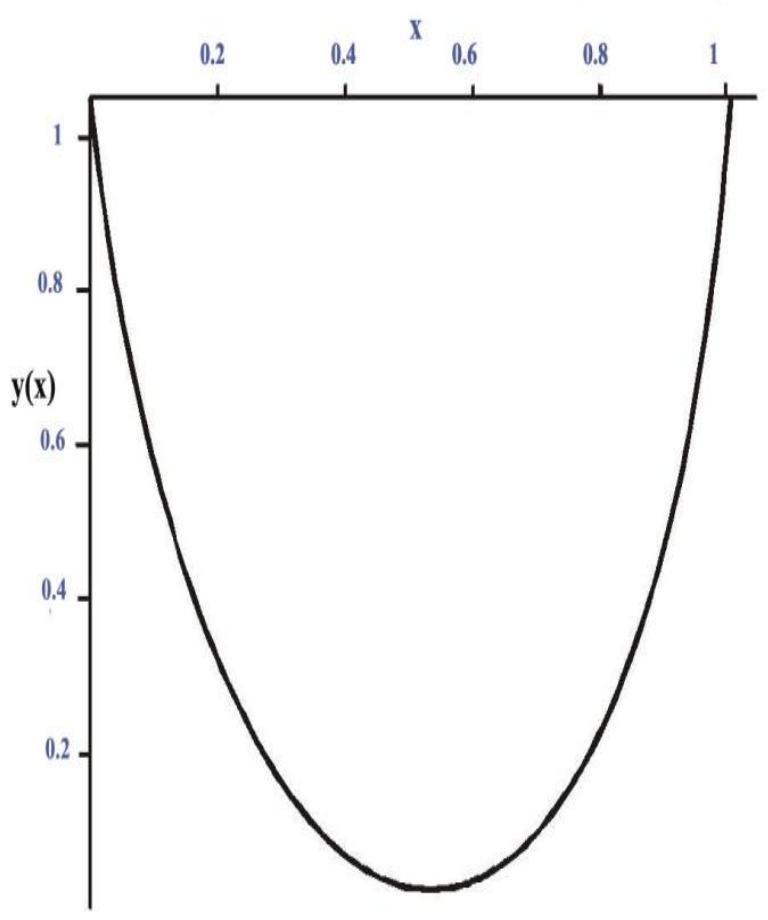

Figure 2(A): Exact Solution 


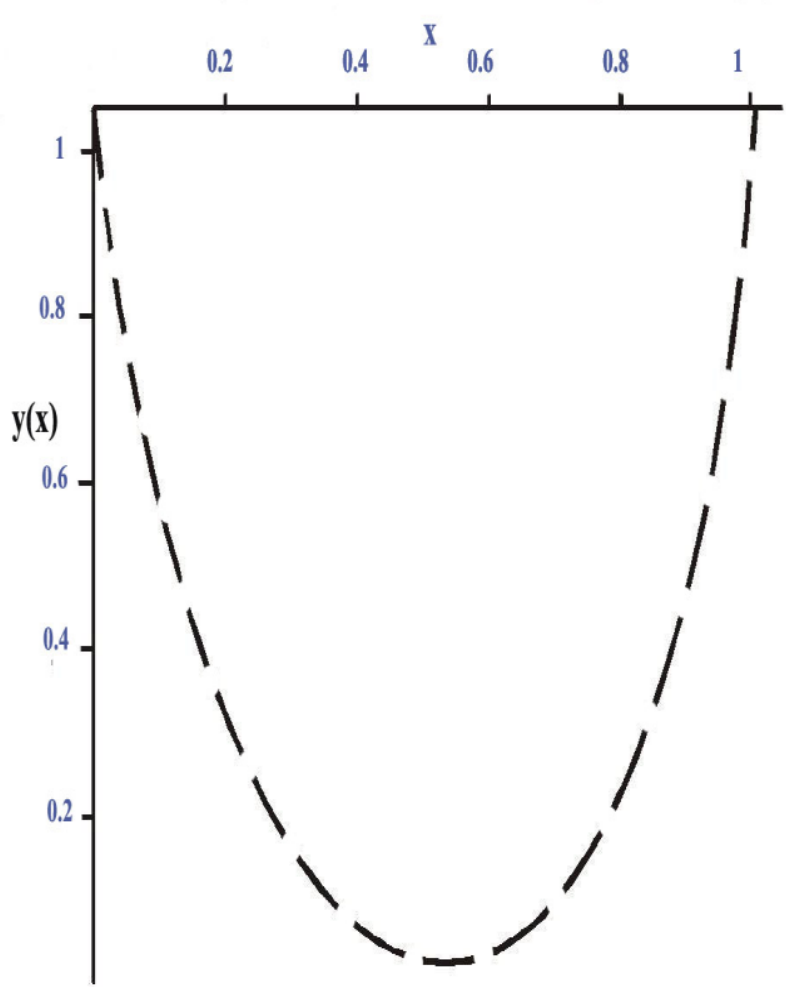

\section{CONCLUSION \& FUTURE SCOPE}

This paper represented simple and straight forward numerical technique based on Haar wavelet is proposed for solving the differential equation. This method is simple and has small computation cost and also very convenient for solving variety of boundary value problems. This paper presented numerical solution very close to the exact solution. So Haar wavelet method is very simple, fast and reliable. It is observed that Haar Wavelet method can be extended for more collocation points. Thus it will be interesting to study for what class of differential equations; the Haar wavelet will give better results.

\section{REFERENCES}

[1] Stromberg, J.O., 1981. In Proceedings of Harmonic Analysis, University of Chicago, 475-494.

[2] Grossmann, A., and Morlet, J., 1984. SIAM J.Math.Anal. 15,723 .

[3] Meyer, Y., 1989. Analysis at Urbana 1: Analysis in Function Spaces Cambridge University Press, Cambridge.

[4] Mallat, S.G., 1989. Trans. Amer.Math.Soc.315,69.
[5] Daubechies, I., 1988. "Orthonormal bases of compactly supported wavelets”, Comm. Pure Appl. Math., 909-996.

[6] Strang, G., 1989. SIAM Rev. 31, 614.

[7] Hsiao, C.H., 2004. "Haar wavelet approach to linear stiff systems", Mathematics and Computers in Simulation, 561567.

[8] Daubechies, I., 1992. Ten Lectures on Wavelets (SIAM, Philadelphia,)

[9] Chui, C.K., 1992. "An introduction to wavelets" (Academic Press, Boston, MA).

[10] Lepik, ULO., 2005. Numerical solution of differential equations using Haar wavelets, Mathematics and Computers in Simulation, 127-143.

[11] Daubechies, I., 1992. "Ten Lectures on Wavelets, SIAM, Philadelphia, PA".

[12] Kaiser, G., 1994. "A friendly guide to wavelets," Boston: Birkhauser.

[13] Lepik, U., 2007. "Numerical Solution of Evolution Equations" by the Haar Wavelet Method. Appl. Math. and Comput., 185, 695-704.

[14] Chen, C.F., and Hsiao, C.H., 1997. "Haar Wavelet Method for Solving Lumped and Distributed Parameter Systems," IEE Proc, Number 144 in Control Theory Appl., 87-94.

[15] Hsiao, C.H., 1997. "State Analysis of Linear Time Delayed Systems via Haar Wavelets," Math. Comp. Simulat., 44: 457-470.

[16] Cattani, C., 2004. "Haar Wavelets Based Technique in Evolution Problems," Proc. Estonian Acad. Set Phys. Math., 1: 45-63.

[17] Siddiqui, Abul Hasan., 2003. "Wavelet Method for Partial Differential Equations and Image Processing, Numerical Methods," Wavelet Methods and Image Processing ISBN: 978-0-8247-4097-9, ISBN: 978-0-203-91301-7, doi: 10.1201/97802039 13017. Ch 11.

[18] Hariharan, G., 2013. "An Overview of Haar Wavelet Method for Solving Differential and Integral Equations," World Applied Sciences Journal, 23, 01-14

[19] Haar, A., 1910. "Zur Theorie Der Orthogonalen Funktionsysteme," Math. Ann. 69, 331-371.

[20] Hsiao, C.H. and S.P. Wu, 2007 "Numerical Solution of Time-Varying Functional Differential Equations" via Haar Wavelets. Appl. Math. Comput., 188 (1): 1049-1058. 